\title{
EXPLOITING REFERENCE IMAGES IN EXPOSING GEOMETRICAL DisTORTIONS
}

\author{
Rimba Whidiana Ciptasari ${ }^{1,2}$ and Kouichi Sakurai ${ }^{1}$ \\ ${ }^{1}$ Department of Informatics, Kyushu University, Fukuoka, Japan \\ sakuraieinf.kyushu-u.ac.jp \\ ${ }^{2}$ Faculty of Informatics, Telkom Institute of Technology, Bandung, Indonesia \\ rimba@itslab.inf.kyushu-u.ac.jp
}

\begin{abstract}
Nowadays, image alteration in the mainstream media has become common. The degree of manipulation is facilitated by image editing software. In the past two decades the number indicating manipulation of images rapidly grows. Hence, there are many outstanding images which have no provenance information or certainty of authenticity. Therefore, constructing a scientific and automatic way for evaluating image authenticity is an important task, which is the aim of this paper. In spite of having outstanding performance, all the image forensics schemes developed so far have not provided verifiable information about source of tampering. This paper aims to propose a different kind of scheme, by exploiting a group of similar images, to verify the source of tampering. First, we define our definition with regard to tampered image. The distinctive features are obtained by exploiting Scale-Invariant Feature Transform (SIFT) technique. We then proposed clustering technique to identify the tampered region based on distinctive keypoints. In contrast to $k$-means algorithm, our technique does not require the initialization of $k$ value. The experimental results over and beyond the dataset indicate the efficacy of our proposed scheme.
\end{abstract}

\section{KEYWORDS}

The trace transform, Geometrical transformation, Reference images, Clustering technique, Image correlation

\section{INTRODUCTION}

\subsection{Background}

In general, the main purpose of computer forensics is the proper identification, extraction, documentation, and interpretation of computer data to determine potential legal evidence. When we bring this definition into the digital image forensic's point of view [1], recovering information on the history of an image could be part of evidence collection. We need evidence to prove that someone has manipulated the photograph. Among several types of evidence discussed by Solomon et al. in [2], we observe that finding the traces of forgeries could fall into demonstrative evidence. It exploits visual aids or other illustrations to give the explanation on some of the more technical details of the evidence. Inspired by such evidence, identifying the source of tampering grows to be our subject of interest.

\subsection{Prior Work in Tampering Detection}

When dealing with high-quality image forgery creation, numerous ways of image processing are exploited. The image can be tampered at varying degrees by employing sophisticated editing software, including changing the colour of object, blurring out the object, or changing on the weather condition. Image splicing, one of the most common types of manipulation, involves DOI : 10.5121/ijma.2013.5301 
The International Journal of Multimedia \& Its Applications (IJMA) Vol.5, No.3, June 2013

copying a certain small portion from an image and pasting onto another image. In order to produce convinced tampering, geometrical transformations are often performed. It is consequently, necessary to construct a scheme which is able to detect and expose such transformed regions.

Researchers have devised various techniques, which can be classified into two main categories: image as the source of tampering identification and image tampering classification.

\subsubsection{Image as source of tampering}

A number of sources of tampering identification schemes which belong to the duplicated region have exhibited promising results. Ryu et al.[3] calculated the magnitude of Zernike moments of overlapping pixel-blocks to produce rotated region feature vectors. The duplicated regions were then detected by lexicographically sorting these feature vectors. However, scaled region identification was not addressed in the paper. $\mathrm{Li}$ and $\mathrm{Yu}$ [4] proposed a scheme based on FourierMellin where significant rotation invariance was achieved by taking projection along radius directions. Nevertheless, the scale invariance seems to be valid only over a small range. Solorio and Nandi [5] employed log-polar block descriptors to detect rotated, scaled and reflected regions. Nevertheless, the false alarms are introduced when the scheme is employed to images containing large textured regions. Numerous schemes using scale-invariant feature transform (SIFT) features have been proposed in [6][7][8] to handle various transformations. These features are not robust to many post-processing operations such as blurring and flipping. Thus, Kakar and Sudha [9] proposed feature's computation improvement, which is robust to such operations. The scheme, however, seems to be robust only under affine transformations.

Apart from the issue of the duplicated region, detecting the traces of forgeries making use of estimation-based approach has been developed. Popescu and Farid [10] exploited expectation/maximization (EM) algorithm to detect re-sampling's lattice of the original image. Unlike Popescu and Farid, Prasad and Ramakrishnan [11] have a propensity to investigate the properties of a re-sampled discrete sequence and proposed deterministic techniques to detect resampling. Ye et al. [12] investigated blocking artifacts introduced during JPEG compression. The inconsistencies caused by compression could be used as evidence of image integrity.

\subsubsection{Image tampering classification}

Other approaches to detect tampered image are based on a machine learning framework. Farid and Lyu [13] built a classification scheme to differentiate between natural image and tampered image. $\mathrm{Ng}$ et al. [14] improved the performance of bicoherence features [15] to detect spliced image. Avcibas et al. [16] constructed a classifier by employing image quality metrics as the essential features. The rationale of using this metrics is to examine different quality aspects of an image impacted during manipulations. Bayram et al. [17] exploited the feature correlations between bit planes and binary texture characteristics within the bit planes. Chen et al.[18] extracted the image features by calculating the moment of wavelet characteristics functions and phase congruency. Dong et al. [19] analyzed the spliced artifact on image run-length representation and edge statistics. Sutthiwan et al. [20] employed support vector machine (SVM) to train image features as well. The image model is based on Markovian Rake Transform (MRT) on image luminance.

\subsection{Key Contribution}

Regarding demonstrative evidence discussed in preceding section, both estimation-based and machine learning-based approaches do not directly indicate the source of tampering. In case of duplicated region detection, the tampered region is originally derived from the same image, and the scheme is seemingly able to identify the distorted region. Apart from this issue, we are moderately interested in analyzing the suspicious regions derived from other different images. 
The International Journal of Multimedia \& Its Applications (IJMA) Vol.5, No.3, June 2013

An outstanding and similar work involving grouping of images was recently proposed by Rosa et al.[21]. They investigated on finding the dependencies among images representing the same real scene, and then constructed a sort of graph describing on how these images have been generated and how the information about the real scene contained in such images has changed. We highlight two key points on their scheme. Firstly, their scheme focused on multi images rather than a single image. In a certain case, it is difficult to employ their scheme to authenticate image validity. Second, they did not involve the scenario that the image could be generated from two different images as well as the image forgery scenario. Though we certainly concentrate on a single image, we propose comparatively different scheme involving a group of similar images, in terms of colour, texture, or shape, to the target image. The key contributions are highlighted as follows:

1) Non-blind recovery scheme (NBR-s). To the best our knowledge, our scheme to be the first concrete technique towards appropriate tools which exploits a group of reference images to identify the source of tampering. The resulting source of tampering could be taken as evidence that some form of manipulation occurred.

2) Region extraction based on clustering algorithm. In previous work ([22][23]), the spliced artifact identification relies on edge detection technique. Due to this condition, the images which do not introduce any edges are hard to be detected, or might cause fault extraction. The proposed approach introduces clustering algorithm to group the distinctive keypoints with regard to the tampered region. Unlike $k$-means clustering, the algorithm automatically produces the number of cluster during the process.

3) Complex dataset of tampered images. We construct a dataset to be used for tampering detection consisting of 344 images employing various transformations. A realistic dataset of alleged forgery images has been also considered for testing purposes.

The rest of the paper is structured as follows: in section 2, we give a mathematical formalization of the concept of non-blind recovery scheme. In section 3, we describe the overall process of geometric tampering detection and methods used in our scheme. Some experimental results consider both synthesized images and realistic dataset are discussed in section 4 . Finally section 5 concludes the paper with summary and a research direction.

\section{Problem Formalization}

We begin with our definition on a suspected spliced-image formally defined as follows.

\section{Definition 1 (suspected spliced-image).}

Suppose there are $n$ suspicious regions $\boldsymbol{T}=\left\{t_{1}, t_{2}, \ldots, t_{\mathrm{n}}\right\}$ derived from a given image $I$, and $m$ reference images $\boldsymbol{R}=\left\{r_{1}, r_{2}, \ldots, r_{m}\right\}$. Assume that there exists at least one $t_{i}$ such that $t_{i} \subseteq r_{j}$ for $i=1, \ldots, n$ and $j=1, \ldots, m$. Then, image $I$ is said to be $a$ suspected spliced-image.

Furthermore, given two set of gray-scale images $\mathrm{I}_{A}$ which refers to authentic images and $\mathrm{I}_{T}$ represents tampered images, where an image $I \in\left(\mathrm{I}_{A} \cup \mathrm{I}_{T}\right)$ is an $M \times N$ matrix, whose entries are integer values $\in[0,255]$. Suppose there exist a large image database $\Delta \mathrm{B}$ consisting of registered authentic images defined as $\Delta \mathrm{B}=\left\{a_{1}, a_{2}, \ldots, a_{n}\right\}$. An image is said to be authentic if it is directly derived from image acquisition device or it has not undergone some image processing functions. We consider an image retrieval system (IRS) $\Phi_{f}$ consisting of a set of properties $\varphi_{f}$ characterizing the IRS i.e. color, shape and texture properties. IRS is a system to retrieve images which is similar to a user-defined specification or pattern. The IRS is described as $\Phi_{f}():. I \times \varphi_{f} \times \Delta \mathrm{B} \rightarrow \boldsymbol{R}$, where each element of images $\boldsymbol{R} \in \mathrm{I}_{A}$ and $\boldsymbol{R}$ is regarded as reference images. 
The International Journal of Multimedia \& Its Applications (IJMA) Vol.5, No.3, June 2013

\subsection{Suspicious region extraction}

The NBR-s consists of two main processes namely suspicious region extraction and geometrical parameter estimation. Suppose there exist an image $I$ belong to $\mathrm{I}_{T}$, and $\mathrm{P}$ is an image belong to $\boldsymbol{R}$. By assuming that $I$ contains small portion of $\mathrm{P}$, our interest is to identify the relative position of small subset $\mathrm{P}$ located on $I$ by extracting the distinctive keypoints between such images. Suppose there exist set of keypoints $\mathbf{X}=\left\{X_{I}, X_{R}\right\}$ where $X=\left\{\mathrm{x}_{1}, \ldots, \mathrm{x}_{\mathrm{n}}\right\}$ and set of descriptors $\mathbf{D}=\left\{D_{I}, D_{R}\right\}$ where $D=\left\{\operatorname{desc}_{1}, \ldots, \operatorname{desc}_{n}\right\}$. The best candidate matched keypoint in $\mathbf{X}$ is attained by computing Euclidian distance between their descriptors in $\mathbf{D}$. We exploit a more effective measure by using the ratio between the distance of the closest neighbor to that of the second-closest one, and comparing it with a threshold. For sake of clarity, given a keypoint we define a similarity vector $\mathrm{S}$ $=\left\{d_{1}, d_{2}, \ldots, d_{n-1}\right\}$ that represents the sorted euclidean distances with respect to the other descriptors. The matched keypoint is obtained if the following constraint is satisfied: $d_{1} / d_{2}<T$. Iterating on each keypoint in $\mathbf{X}$, we can obtain the set ofcorresponding matched points $\mathbf{K}=\left\{\left(\mathrm{k}_{\mathrm{I}}, \mathrm{k}_{\mathrm{Rj}}\right)\right\}$. Note that there are groups of points on $I$ corresponding to ones on $\mathrm{P}$. To identify the suspicious regions, clustering is then conducted on either groups of $\mathbf{K}$. To finally confirm the suspicious region, we compute the correlation coefficient $c$ between corresponding clusters on $I$ and $\mathrm{P}$ with a suitable threshold $\tau$. if ç is lower (res. higher) than the threshold then the region is deemed as suspicious region (authentic region).

\subsection{Geometrical parameter estimation}

Recall the comparison among corresponding clusters on $I$ and $\mathrm{P}$ in preceding section. Let us denote the set of extracted suspicious region on $I$ selected by threshold $T$ be $\mathbf{S}$ and set of associated region with $\mathbf{S}$ on $\mathbf{P}$ be $\mathbf{E}_{\mathbf{R}}$. In this case, $\mathbf{S}$ and $\mathbf{E}_{\mathbf{R}}$ are regarded as distorted and original region, respectively. Several combinations of appropriate triple feature $\Pi$ of the Trace transform are then computed over $\mathbf{S}$ and $\mathbf{E}_{\mathbf{R}}$. Let $\Pi(\mathrm{F}, \mathrm{C} 1)$ and $\Pi(\mathrm{F}, \mathrm{C} 2)$ be triple feature of $\mathbf{S}$ and $\mathbf{E}_{\mathbf{R}}$, respectively. Rotation angle $\theta$ and scaling factor $s$ are then estimated by simply finding the ratio, and difference between $\Pi(\mathrm{F}, \mathrm{C} 1)$ and $\Pi(\mathrm{F}, \mathrm{C} 2)$, respectively.

\section{Proposed Scheme}

For exposition purpose, we first describe the overview of the proposed scheme. Enlightening the concept of proposed clustering technique and complete description on geometrical parameter estimation are then discussed.

\subsection{System overview}

Figure 1 describes an overview of the general architecture of the system we used to identify the source of geometrical tampering. To eliminate processing time and have meaningful results, we randomly take three images on $\boldsymbol{R}$ as references such that $\mathbf{S} \subset \boldsymbol{R}$. 


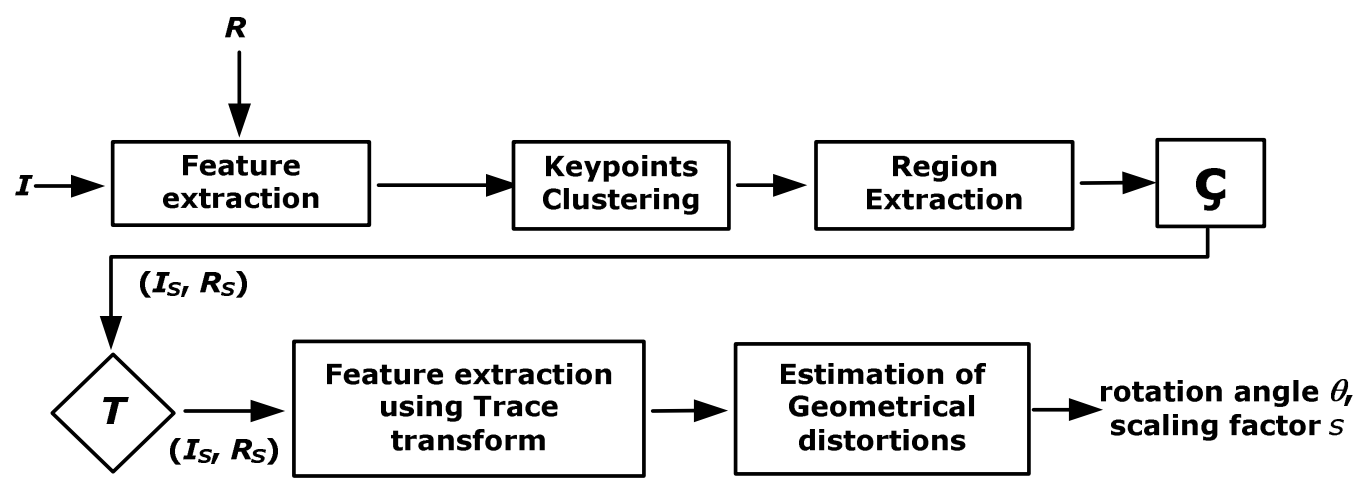

Figure 1.The overall process of verification system architecture. The block denoted by Ç computes the correlation coefficient between suspicious region candidate(s) $I_{S}$ and its similar regions $R_{S} . \tau$ denotes the comparison of ç against the decision threshold.

The verification procedure is conducted as follows. To begin with, IRS is exploited to input image $I$ in order to obtain a group of similar images. The retrieved images are categorized based on three properties, i.e. colour, texture, and moment. Despite the fact that the images have the same properties, it is likely to have a completely different object of interest. Thus, the most representative images are manually selected from these results as reference images $R$.

The first stage in our method is to find image keypoints and collect image features at the detected keypoints on both target $I$ and reference $R$. Our method is based on effective keypoint and feature computation algorithm referred to as Scale Invariant Feature Transform (SIFT) [24]. At each keypoint, a 128-dimensional featurevector is generated from the histograms of local gradientsin its neighborhood. To guarantee the obtained feature vector invariant to rotation and scaling, the size of the neighborhood is determined by the dominant scale of the keypoint, and all gradients within are aligned with the keypoint's dominant orientation[6] To provide the feature vector invariant to local illumination changes, the obtained histograms are then normalized to unit length. Afterward, the detected SIFT keypoints on $I$ and $R$ are matched based on their feature vectors. We consider the suspicious region tends to emerge as a collection of adjacent keypoints. Clustering is, therefore, performed on the matched keypoints to identify the suspicious region. Furthermore, the system calculates the correlation coefficient $\zeta$ between the corresponding detected suspicious regions. To accept or reject whether the region is subjected to suspicious one, ç is compared to a specific threshold $\tau$.

\subsubsection{Keypoint Clustering}

Recall $\mathbf{K}=\left\{\left(\mathrm{k}_{\mathrm{Ii}}, \mathrm{k}_{\mathrm{Rj}}\right)\right\}$ be the set of matched keypoints where $\mathrm{k}_{\mathrm{Ii}}$ and $\mathrm{k}_{\mathrm{Rj}}$ are keypoints located on target and reference images, respectively. Note that they correspond with each other. Next, matched keypoints clustering is accomplished to estimate the suspicious region. In contrast to $k$ means algorithm, we propose a technique that requires no initial $k$ value as the number of clusters. Each cluster is represented by the centre of the cluster and the algorithm converges to stable members of clusters. As clustering is not the main subject, we do not draw the comparison to $k$-means algorithm. The clustering procedure is described as follows.

\footnotetext{
Input : The set of matched keypoints $\mathbf{K}=\left\{\left(\mathrm{k}_{\mathrm{I}}, \mathrm{k}_{\mathrm{Rj}}\right)\right\}$

The distance threshold $r$

Output : The pairs of selected regions $\left(I_{S}, R_{S}\right)$
} 
The International Journal of Multimedia \& Its Applications (IJMA) Vol.5, No.3, June 2013

Step 1. Initialization.

1: We begin with choosing an arbitrary point from $\mathbf{K}$, and we select $k_{I I}$ and $k_{R l}$ as seed points.

2: Assign each keypoints $\left(k_{I}, k_{R}\right) \in \mathbf{K}$ to the cluster with the nearest seed point. The basic idea is to group element of $\mathbf{K}$ based on the distance up to $r$ from current coefficient to the next one (e.g. $d\left(k_{i}, k_{i+1}\right), d\left(k_{i+1}, k_{i+2}\right)$, so on), and is formulated as follows.

$$
\mathbf{D}(k, r)=\{x \in \mathbf{X} \mid d(k, x) \leq r\}
$$

where $k$ is the set of SIFT keypoints, and $r$ is an acceptable distance from $c_{i}$ to $c_{i+1}$ determined experimentally. Consider $k$ is the matched keypoint positioned at $(x, y)$, the squared Euclidian distance is adopted for this purpose denoted as follows:

$$
d\left(q_{i}, q_{i-1}\right)=\sqrt{\left(x_{i}-x_{i-1}\right)^{2}+\left(y_{i}-y_{i-1}\right)^{2}} .
$$

Step 2. Let $\mathbf{C}_{\mathbf{I}}$ and $\mathbf{C}_{\mathbf{R}}$ be the set of clusters located on target and reference image resulting from step 1. Next step is to compute centroids of each cluster and distance of each keypoints $\left(k_{l}, k_{R}\right) \in \mathbf{K}$ to these new centroids.

Step 3. Do re-clustering as described in step 1 . The procedure is repeatedly carried out until the algorithm converges to stable of cluster members.

\subsubsection{Region Extraction}

Recall $\mathbf{C}_{\mathbf{I}}$ and $\mathbf{C}_{\mathbf{R}}$ are the set of obtained clusters from the preceding subsection. The next step is to process these clusters to obtain suspicious regions. We use two different block size, i.e. $16 \times 16$ and $32 \times 32$ pixels, and extract the region centered at each centroid cluster.

In order to properly verify the suspicious region, we compute the normalized cross-correlation (NCC) formulated as

$$
\varsigma(u, v)=\frac{\sum_{x, y}\left[f(x, y)-\bar{f}_{u, v}\right][t(x-u, y-v)-\bar{t}]}{\left\{\sum_{x, y}\left[f(x, y)-\bar{f}_{u, v}\right]^{2} \sum_{x, y}[t(x-u, y-v)-\bar{t}]^{2}\right\}^{0.5}}
$$

where $f$ is the extracted region on reference image, $\bar{t}$ is the mean of the suspicious region on target image, and $\bar{f}_{u, v}$ is the mean of $f(x, y)$ in the region under the suspicious region. The correlation coefficient is in the range $[-1,1]$, with larger value indicating higher level of similarity. $\varsigma$ close to -1 means the matching entities are inverse of each other, $\varsigma$ close to 1 refers to matching entities are exactly the same, and $\varsigma=0$ is an indication of no relationship between the matching entities. To accept or reject whether the extracted regions are regarded as suspicious ones, we compare the correlation coefficient between them with a suitable threshold $\tau$. Considering a lower value of $\tau$ leads to better detection accuracy of suspicious regions, but may increase false positive rate. A higher value of $\tau$ may yield regions that are strongly similar, but could miss detections of regions having weak correlations. We set a default value of $\tau=0.8$ experimentally for a good trade-off between detection accuracy and false detection rate.

\subsubsection{Geometrical Distortions Estimation}

It is required a method suited to construct features that are invariant and/or sensitive to geometric transformation, e.g. rotation, translation, and scaling. We consider trace transform [25] that offers the option to construct features from an image with desirable properties.

Let $\left(I_{S}, R_{S}\right)$ be a pair of extracted region and its corresponding region located in either reference images. A method to represent characteristics of region $I_{S}$ over $R_{S}$ decided by $T(\phi, p)$ is called the 
The International Journal of Multimedia \& Its Applications (IJMA) Vol.5, No.3, June 2013

trace transform as depicted in Figure 2. The trace transform $T(\phi, p)$ is computed by drawing lines denoted by $t$ parameterized by distance $p$ (11 and 21 values from $-p$ to $p$. Note that we evaluate two types of block size, i.e. $16 \times 16$ and $32 \times 32)$ and angle $\phi(240$ values from 0 to $2 \pi)$.

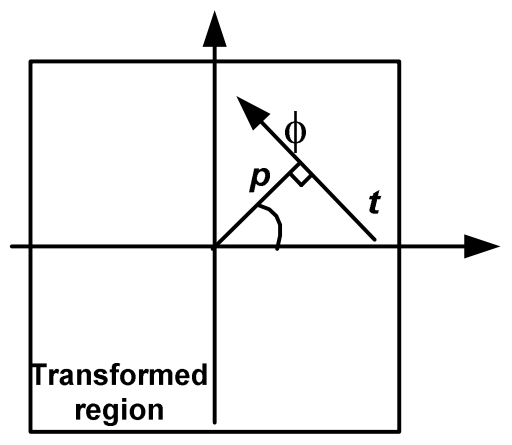

Figure 2. Trace transform parameters

Feature values for calculating the geometrical factors are conducted by computing triple features which consist of trace function $T$, diametric function $P$, and circus function $\Phi$ over transformed regions. In order to produce triple features, we compose combinations selectively from equations in Table 1.

Table 1. Invariant and Sensitive Functional for Triple Feature Construction

IF2: $\left(\int|\xi(t)|^{q} d t\right)^{r}$
IF3: $\int|\xi(t)|^{\prime} d t$
IF4: $\int(t-S F 1)^{2} \xi(t) d t$
IF5: (IF4/IF1) $)^{1 / 2}$
IF9: Amplitude of 2nd harmonic of $\xi(t)$
IF10: Amplitude of 3rd harmonic of $\xi(t)$
SF1: $\int \xi(t) d t / I F 1$
SF5: phase of $2^{\text {nd }}$ harmonic of $\xi(t)$
SF6: phase of $3^{\text {rd }}$ harmonic of $\xi(t)$
SF7: phase of $4^{\text {th }}$ harmonic of $\xi(t)$

\section{Evaluation}

To evaluate the effectiveness of our system, we have constructed a set of generated forgery images with suspicious regions. Further, we present the experimental results and conduct the comparison to the existing schemes.

As a basic assumption we suppose to work with image splicing only that is an image derived by combining image portions from different images without further post-processing such as smoothing of boundaries among different portions.

\subsection{Dataset construction}

Our scenario involves forgery images containing several suspicious regions derived from different images. Distorted images are generated based on 55 images of size $128 \times 128$ provided by DVMM laboratory [26]. Two types of geometric transformation are applied, i.e. rotation and scaling. The selected region is rotated with a random angle $\theta \in\left[0^{\circ}, 360^{\circ}\right]$ and scale up/down with 
a random factor $s \in[0.8,1.0]$. We use two different block sizes, i.e. $16 \times 16$ and $32 \times 32$, for each type of distortion resulting in 344 distorted images. In addition, we exploit spliced images used in [23] to evaluate the scheme effectiveness against non-geometrical transformations. Figure 3 depicts generated tampered images used in our experiments. The distorted images are constructed in the following way:

i. For each authentic image, we first collect several arbitrary images with different scene.

ii. We randomly choose such a region(s) from those images in such a way that it looks natural, copy the region(s), and paste onto the authentic one.

iii. The region is either rotated or scaled prior to pasting onto the authentic image.
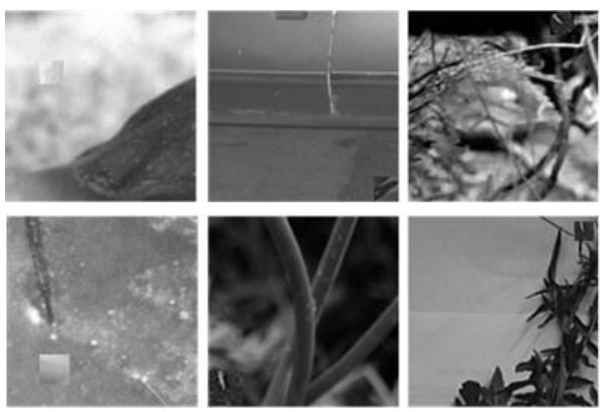

Figure 3. Example of dataset used in the experiment. In the first row from left to right, the tampered region is rotated by angle 91, 181, and 31 respectively. The second row, the tampered region is scaled with factor $1.2,1.4$, and 0.8 , respectively.

\subsection{Clustering Results}

One of the main contributions of this paper is related to the design of clustering algorithm aiming at tampered region identification. The accurateness of our verification scheme relies on the precision of suspicious region extraction. Our first experiment addresses the sensitivity of our method on identifying the suspicious region.

Extraction performance was measured in terms of true positive rate (TPR) and false positive rate (FPR) where TPR is the fraction of tampered images correctly identified as such, while FPR is the fraction of original images that are not correctly identified:

$$
\begin{aligned}
& T P R=\frac{\# \text { images detected as distorted being distorted }}{\# \text { distorted images }} . \\
& F P R=\frac{\# \text { images detected as distorted being original }}{\# \text { original images }} .
\end{aligned}
$$

Suspicious region extraction results obtained by employing the proposed clustering algorithm are shown in Figure 4. To reduce the processing time, we exploit only three reference images related to the input image. As stated earlier in Section 3.1, the reference images shown in Figure 4(b) are manually selected from IRS. The clustering results depicted in Figure 4(c) confirm that the adjacent keypoints representing the suspected region can be roughly grouped. There are several corresponding pairs of extracted regions obtained from a clustering process. As we expect that extracted region shown in the first row of Figure 4(e) indicates the correlation above the threshold that is 0.8174 . Other pairs demonstrate correlation values below the threshold. Thus we may conclude that the given image in Figure 4(a) contains another image's sub-region. In other words, regarding Definition 1 the image is deemed as the tampered one. 
The International Journal of Multimedia \& Its Applications (IJMA) Vol.5, No.3, June 2013

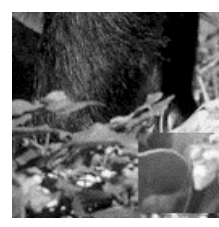

(a)
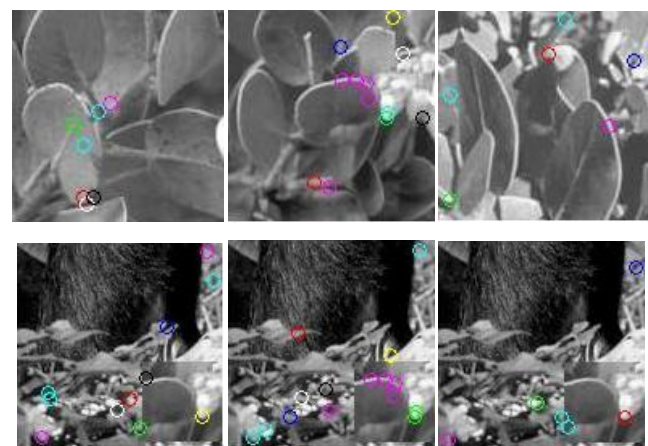

(c)
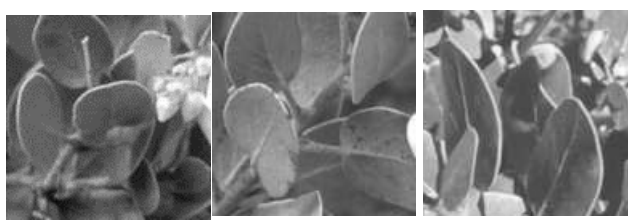

(b)
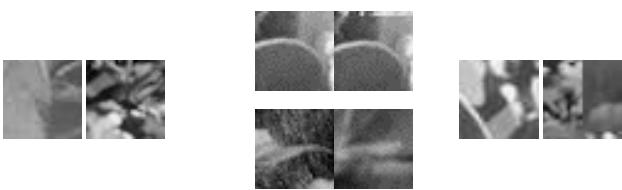

(d)

(e)

Figure 4. Tampered region identification using the proposed clustering technique. (a) An example of image in question. (b) Samples of reference images related to image (a). (c) Corresponding clustering results on both reference and input images. (d) - (e) Region extraction results that correspond to each reference image, respectively.

Table 2 reports the diagnostic parameters dealing with suspicious region extraction. In terms of rotation, for block size of $16 \times 16$ and $32 \times 32$, our method achieves TPR greater than $88 \%$, with FPR around $11 \%$. Similarly, in case of scaling the proposed method attain TPR approximately $90.97 \%$ on average with FPR around $15 \%$. Compared to previous work in [23] the proposed approach indicates an improvement on detection accuracy increased by $0,57 \%$.

Table 2. TPR and FPR values (in percentage) for rotation and scaling with respect to suspicious region extraction

\begin{tabular}{|c|c|c|c|c|c|c|}
\hline & \multicolumn{2}{|c|}{ Rotation } & \multicolumn{2}{c|}{ Scaling } & \multirow{2}{*}{ Non-geometric } & $\begin{array}{c}\text { Non-geometric } \\
\text { Ciptasari et al.[23] }\end{array}$ \\
\cline { 2 - 5 } & $16 \times 16$ & $32 \times 32$ & $16 \times 16$ & $32 \times 32$ & & 92,08 \\
\hline TPR (\%) & 88,75 & 88 & 94,4 & 87,5 & 95,32 & 2,22 \\
\hline FPR (\%) & 10 & 11,25 & 28 & 3,7 & 1,81 & \\
\hline
\end{tabular}

\subsection{Tampering Detection Performance}

To demonstrate the efficacy of the proposed approach, tampering detection caused by geometrical distortions is included for completeness and to experimentally show that a clustering step is fundamental to obtain satisfactory results. The final step of the proposed approach is to verify whether the image has undergone some geometrical distortions, i.e. the localization of the image regions that have been modified for malicious purposes.

To estimate the geometrical parameters, we evaluate approximately 25 triple feature combinations and experimentally choice the appropriate ones as listed in Table 3 . To recover the scaling factor between two images, we need to calculate a certain triple feature for the two images and take the ratio of these values [25]. In addition, the rotation angle is identified by simply finding the difference between the values of a triple feature calculated for two versions of the same image that are rotated with respect to each other[25]. We adopt these techniques by 
The International Journal of Multimedia \& Its Applications (IJMA) Vol.5, No.3, June 2013

calculating the extracted suspicious regions resulting from preceding sub-section instead of the whole image areas.

Table 3 Triple Feature Combinations with respect to rotation and scaling

\begin{tabular}{|c|c|c|c|c|c|c|c|c|c|c|}
\hline & \multicolumn{10}{|c|}{ TRIPLE FEATURE COMBINATIONS } \\
\hline Rotation & $\begin{array}{l}\mathrm{T}=\mathrm{IF} 4, \\
\mathrm{P}=\mathrm{IF} 10, \\
\mathrm{C}=\mathrm{SF} 6\end{array}$ & $\begin{array}{l}\mathrm{T}=\mathrm{IFC} \\
\mathrm{P}=\mathrm{SF} \\
\mathrm{C}=\mathrm{SF}\end{array}$ & $\begin{array}{l}\mathrm{T}=\mathrm{IF} 3, \\
\mathrm{P}=\mathrm{IF6}, \\
\mathrm{C}=\mathrm{SF} 7\end{array}$ & & & $\begin{array}{l}\mathrm{T}=\mathrm{IF} 2, \\
\mathrm{P}=\mathrm{SF} 1, \\
\mathrm{C}=\mathrm{SF} 5\end{array}$ & $\begin{array}{l}\mathrm{T}=\mathrm{IF} 3, \\
\mathrm{P}=\mathrm{SF} 1, \\
\mathrm{C}=\mathrm{SF} 5\end{array}$ & & $\begin{array}{l}\text { IF4, } \\
\text { SF1, } \\
\text { SF5 }\end{array}$ & $\begin{array}{l}\mathrm{T}=\mathrm{IF} 10, \\
\mathrm{P}=\mathrm{IF6}, \\
\mathrm{C}=\mathrm{SF5}\end{array}$ \\
\hline Scaling & \multicolumn{2}{|c|}{$\begin{array}{c}\mathrm{T}=\mathrm{IF} 2 \mathrm{a}, \\
\mathrm{P}=\mathrm{SF} 1, \mathrm{C}=\mathrm{IF} 3\end{array}$} & \multicolumn{2}{|c|}{$\begin{array}{c}\mathrm{T}=\mathrm{IF} 4, \mathrm{P}=\mathrm{SF} 1, \\
\mathrm{C}=\mathrm{IF} 2 \mathrm{a}\end{array}$} & \multicolumn{2}{|c|}{$\begin{array}{c}\mathrm{T}=\mathrm{IF} 5, \mathrm{P}=\mathrm{SF} 1, \\
\mathrm{C}=\mathrm{IF} 6\end{array}$} & \multicolumn{2}{|c|}{$\begin{array}{c}\mathrm{T}=\mathrm{IF} 2 \mathrm{a}, \\
\mathrm{P}=\mathrm{SF} 1, \mathrm{C}=\mathrm{IF} 1\end{array}$} & \multicolumn{2}{|c|}{$\begin{array}{c}\mathrm{T}=\mathrm{IF} 2 \mathrm{a}, \\
\mathrm{P}=\mathrm{SF} 1, \mathrm{C}=\mathrm{IF} 6\end{array}$} \\
\hline
\end{tabular}

Therefore, we begin with evaluating the proposed scheme on extracting the image regions containing geometrical distortions. Figure 5 demonstrates the results against rotation and scaling by exploiting clustering technique. The transparent regions depicted in Figure 5(d) and Figure $5(\mathrm{e})$ indicates the pair of detected regions positioned on target and reference images. In terms of rotation and scaling, each pair produces correlation values greater than the threshold subjected to the first reference in Figure 5(c), i.e. 0.8438 and 0.92966, respectively. As it indicates strong correlation, regarding Definition 1, the image in Figure 5(a) is deemed as the tampered one. Although the keypoints can be detected on the other two references, our method produces no detected regions that fulfil the threshold.

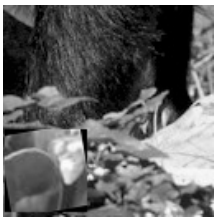

(a)

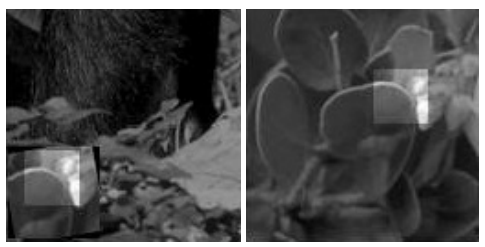

(d)

(b)
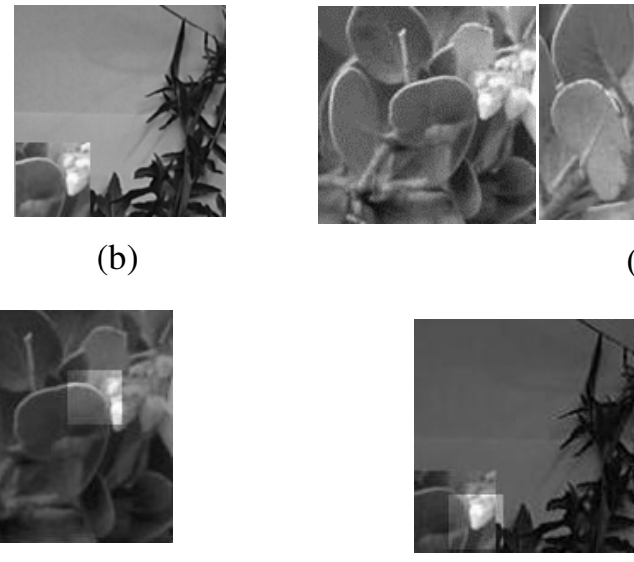

(e)

(c)
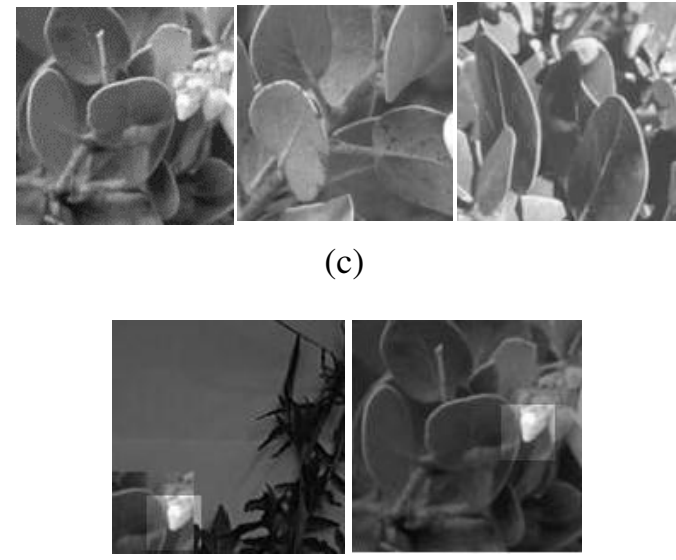

Figure 5. Detection results on forgery images with a region have undergone different type of distortions. (a) The image contains rotated region of 10 degrees. (b) The image contains region scaled down to 0.9. (c) Related reference images to both (a) and (b). The detected regions subjected to rotation and scaling are depicted in (d) and (e), respectively.

We proceed further to estimate the geometrical parameter with respect to different transformations. Table 4 show the results obtained in terms of rotation and scale estimation through exploiting triple feature combinations listed in Table 3. Although FPR values of various transformations ranged from $7 \%$ to $14 \%$, the proposed approach still attains promising results, on average, by $88.59 \%$ of TPR. 
The International Journal of Multimedia \& Its Applications (IJMA) Vol.5, No.3, June 2013

Table 4 TPR and FPR values (in percentage) for rotation and scaling with respect to geometrical parameter estimation

\begin{tabular}{|c|c|c|c|c|}
\hline \multirow{2}{*}{} & \multicolumn{2}{|c|}{ Rotation } & \multicolumn{2}{c|}{ Scaling } \\
\cline { 2 - 5 } & $16 \times 16$ & $32 \times 32$ & $16 \times 16$ & $32 \times 32$ \\
\hline \hline TPR (\%) & 83.64 & 90.91 & 94.12 & 85.71 \\
\hline FPR (\%) & 14.28 & 11.11 & 14.29 & 7.69 \\
\hline
\end{tabular}

Table 5. Average Estimation Error

\begin{tabular}{|l|c|c|}
\hline & Rotation & Scaling \\
\hline \hline Battiato et al.[7] & 1.4762 & 0.04645 \\
\hline Ciptasari et al. [23] & - & - \\
\hline Proposed approach & 1.0961 & 0.0433 \\
\hline
\end{tabular}

Further, to verify the effectiveness of the proposed scheme, we draw the comparison to approaches introduced in [7] and [23]. In contrast to our subject, Battiato et al. [7] principally addressed duplicated region, while the work in [23] did not involve geometric transformation. Therefore, the comparison is conducted in terms of mean absolute error (MAE) values. The results are reported in Table 5. It confirms that the proposed approach obtains a considerable gain both in terms of rotational and scaling accuracy.

\subsection{Realistic detection}

To demonstrate the effectiveness of the proposed scheme from practical point of view, we conduct evaluation against alleged forgery images that has raised public's attention provided by Fourandsix Technologies, Inc [27]. In this section, we highlight our improvement over the scheme proposed in [23]. The improvement result is illustrated in Figure 6. We may confirm that the proposed work precisely extracts the suspected region depicted in Figure 6(d) compared to an extracted region shown in Figure 6(c). It shows that the localized region has undergone geometrical transformation for a malicious purpose.

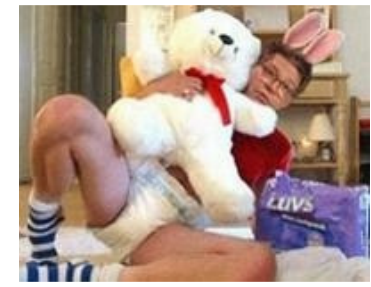

(a)

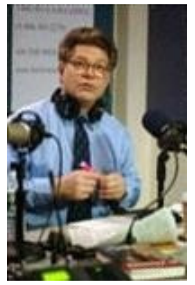

(b)

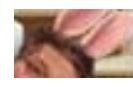

(c)

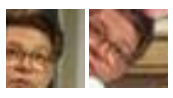

(d)

Figure 6. Improvement result over the previous work in [23] (a) Image in question. (b) A reference image that corresponds to (a). The previous result is depicted in (c), and the improvement detected regions are shown in (d). 
The International Journal of Multimedia \& Its Applications (IJMA) Vol.5, No.3, June 2013

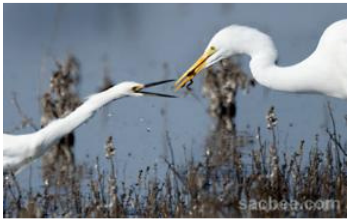

(a)

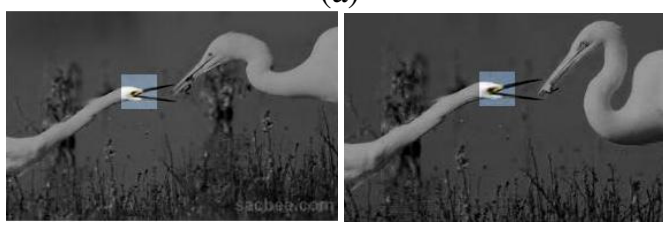

(c)

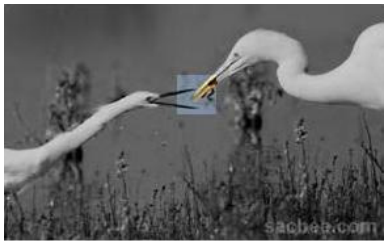

(e)

Figure 7. Verification results over realistic forgery image. (a) Image in question. (b) The reference images with respect to (a). The examples of detected regions found on both first and second references are provided in (c) and (d), respectively.

We provide another result regarding realistic forgery detection in Figure 7 . The suspected regions are approximately detected indicated by transparent regions. Note that the provided images might have undergone some convinced effects by using state-of-the-art image retouching algorithms and tools, thereby causing the correlation coefficient to decline. The detected regions, however, exhibit strong correlation to their corresponding ones with values achieves 0.86348 and 0.89564 .

\section{Concluding Remark}

Considering the objective of computer forensic in offering appropriate collection of digital evidence, designing verification scheme providing a proper proof when a photograph is deemed as a forgery image has attracted our attention. We consider the tampered area to be the localization of image regions that have been modified for malicious purposes. Once the localization region can be confirmed, it might be considered as scientific evidence. We have demonstrated the effectiveness of our proposed scheme in a series of experiments throughout and beyond the dataset.

Though having accomplished promising results in verifying the source of tampering by exploiting the reference images, our method relies on correlation coefficient computation. Figure 7(e) shows that another pair of the tampered regions can be detected. However, as these regions have undergone some manipulations, their correlation is somewhat far behind the threshold, which is 0.48915 .

As a future work, we should concern a more in a dept-analysis to provide a reliable model of reference images to overcome aforementioned limitation. To conclude, we believe, however, that this technique still provide usefulness in digital forensics environment, for example in case of providing proofs of tampering in the court of law. 
The International Journal of Multimedia \& Its Applications (IJMA) Vol.5, No.3, June 2013

\section{ACKNOWLEDGEMENTS}

Research support for the first author is provided by the Directorate General of Higher Education, Ministry of National Education, Indonesia.

\section{REFERENCES}

[1] Redi J.A., Taktak W., Dugelay J.L. (2011) "Digital image forensics: a booklet for beginners", Multimedia Tools and Application, Vol. 51, pp.133-162.

[2] Solomon M.G., Barret D., Broom N.(2005) 'Computer Forensics: Jumpstarts', pp.51 - 57. SYBEX Inc., California

[3] Ryu S.J., Lee M.J., Lee H.K. (2010) "Detection of Copy-Rotate-Move Forgery Using Zernike Moments". In: Information Hiding Lecture Notes in Computer Science, Volume 6387, pp. 51-65

[4] Li W., Yu N.(2010) "Rotation robust detection of copy-move forgery",17th IEEE International Conference on Image Processing (ICIP), pp. 2113 - 2116.

[5] Solorio S.B., Nandi A.K.(2011) "Automated detection and localisation of duplicated regions affected by reflection, rotation and scaling in image forensics", Signal Processing, Elsevier, pp.1759-1770.

[6] Pan X., Lyu S.(2010) "Region Duplication Detection using Image Feature Matching", IEEE Transaction on Information Forensics and Security, 5(4):857-867.

[7] Battiato S., Farinella G.M., Messina E., Puglisi G.(2012) "Robust Image Alignment for Tampering Detection", IEEE transaction on Information Forensics and Security, volume 7, issue 4, pp. 1105 1117.

[8] Amerini I, Ballan L, Caldelli R, Del Bimbo A, Serra G.(2011) “A SIFT-Based Forensic Method for Copy-Move Attack Detection and Transformation Recovery", IEEE transaction on Information Forensics and Security, Volume 6, issue 3, pp. 1099 -1110.

[9] Kakar P., Sudha N.(2012) "Exposing Postprocessed Copy-Paste Forgeries through TransformInvariant Features", IEEE Transaction on Information Forensics and Security, Vol.7 Issue 3, pp.10181028.

[10] Popescu A.C., Farid H.(2005) "Exposing Digital Forgeries by Detecting Traces of Re-sampling", IEEE Transaction on Signal Processing, 53(2):758-767.

[11] Prasad S., Ramakrishnan K.R.(2006) "On Resampling Detection And Its Application To Detect Image Tampering", IEEE International Conference on Multimedia and Expo (ICME).

[12] Ye S., Sun Q., Chang E.C.(2007) "Detecting Digital Image Forgeries by Measuring Inconsistencies of Blocking Artifact", IEEE International Conference on Multimedia and Expo (ICME)

[13] Farid H., Lyu S.(2003) "Higher-order Wavelet Statistics and their Application to Digital Forensics", IEEE Workshop on Statistical Analysis in Computer Vision (in conjunction with CVPR).

[14] Ng T.T., Chang S.F., Sun Q.(2004) "Blind Detection of Photomontage using Higher Order Statistics", IEEE International Symposium on Circuits and Systems, vol. 5, pp. 688 - 691.

[15] Farid H.(1999)“Detecting Digital Forgeries Using Bispectral Analysis”, Technical Report AIM-1657, AI Lab, Massachusetts Institute of Technology.

[16] Avcibas I., Bayram S., Memon N., Sankur B., Ramkumar M.(2004) “A Classifier Design for Detecting Image Manipulations”, IEEE International Conference on Image Processing (ICIP).

[17] Bayram S., Avcibas I., Sankur B., Memon N.(2006) "Image Manipulation Detection", Journal of Electronic Imaging, SPIE and IST, 15(4), 041102.

[18] Chen W., Shi Y.Q., Su W.(2007) "Image Splicing Detection using 2-D Phase Congruency and Statistical Moments of Characteristic Function”, Society of Photo-Optical Instrumentation Engineers (SPIE) Conference Series, vol. 6505, art. No. 65050R. SPIE, Washington.

[19] Dong J., Wang W., Tan T., Shi Y.Q.(2009) "Run-Length and Edge Statistics Based Approach for Image Splicing Detection", International Workshop on Digital Watermarking (IWDW), LNCS 5450, pp. 76-87.

[20] Sutthiwan P., Shi Y.Q., Zhao H., Ng T.T., Su W.(2011) "Markovian Rake Transform for Digital Image Tampering Detection", Y.Q.Shi (Ed.): Transaction on DHMS VI, LNCS 6730, pp. 1-17. Springer, Heidelberg.

[21] Rosa A.D., Uccheddu F., Costanzo A., Piva A., Barni M.(2010) "Exploring Image Dependencies: a New Challenge in Image Forensics", Media Forensics and Security II, edited by Nasir D.Memon, Jana Dittman, Adnan M. Alattar, Edward J. Delp III, Proceeding of SPIE-IS \&\&T Electronic Imaging, SPIE, Vol. 7541, 75410X. 
The International Journal of Multimedia \& Its Applications (IJMA) Vol.5, No.3, June 2013

[22] Ciptasari R.W., Rhee K.H., Sakurai K.(2012) “An Image Splicing Detection based on Interpolation Analysis”, W. Lin, D. Xu, A. Ho, J. Wu, Y. He, J. Cai, M. Kankanhalli, M.-T. Sun,Editors. PacificRim Conference on Multimedia (PCM 2012), LNCS 7674, pp.390-401, Springer, Heidelberg.

[23] Ciptasari R.W., Rhee K.H., Sakurai K.(2013) "Image Splicing Verification based on Pixel-based Alignment Method", The 11th International Workshop on Digital-forensics and Watermarking (IWDW 2012), LNCS 7809, pp. 198-212, Springer, Heidelberg.

[24] D.G. Lowe, (2004) "Distinctive image features from scale-invariant keypoints", Int. J. Computer Vision, vol. 60, no. 2, pp. $91-110$

[25] Kadyrov A., Petrou M.(2001) “The trace transform and its applications”, IEEE Transaction on Pattern Analysis and Machine Intelligence, vol. 23, no. 8, pp. 811-828.

[26] Ng, T.T., Chang, S., Sun, Q.(2004) "A data set of authentic and spliced image blocks", ADVENT Technical Report 203-2004-3, Columbia University, http://www.ee.columbia.edu/trustfoto.

[27] Fourandsix Technologies, Inc. http://www.fourandsix.com/photo-tampering-history/.

\section{Authors}

Rimba Whidiana Ciptasari received her M.Eng degree in software engineering from Bandung Institute of Technology (ITB), Indonesia, in 2005. She is currently pursuing $\mathrm{Ph} . \mathrm{D}$ degree in Graduate School of Information Science and Electrical Engineering, Department of Informatics, Information Technology and Multimedia Security Laboratory, Kyushu University. She is also a faculty member at Telkom Institute of Technology, Indonesia. Her research interest includes information hiding, image processing, and multimedia security.

Kouichi Sakurai is Professor of Department of Computer Science and Communication Engineering, Kyushu University, Japan since 2002. He received B.E., M.E., and D.E. of Mathematics, Applied Mathematics and Computer Science from Kyushu University in 1982, 1986, and 1993, respectively. He is interested in cryptography and information security. He is a member of IPSJ, IEEE and ACM.
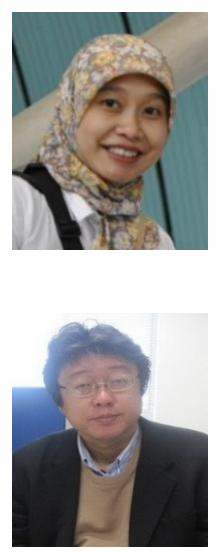